\title{
Do preferences and beliefs in dilemma games exhibit complementarity?
}

I. Martínez-Martínez, J. Denolf, and A. Barque-Duran QI15 


\section{Introduction}

- Start from "Preferences and beliefs in a sequential social dilemma: a within-subjects analysis." (Blanco, et.al., 2014)

- Results seemed promising for a quantum model 


\section{Introduction}

- Start from "Preferences and beliefs in a sequential social dilemma: a within-subjects analysis." (Blanco, et.al., 2014)

- Results seemed promising for a quantum model

- Work in progress. Some problems, advice needed! 
(1) Introduction

(2) The experiment

- The game

- The experiment

(3) Three effects

- FM and SM correlation

- Consensus effect

- Reasoned player

(4) QP\&B model

- The measurements

- Three effects revisited

- Dimension of the belief basis?

- QP\&B model

(5) Discussion and future plans 


\section{The game}




\section{The game}

- Prisoner Dilemma variant, 2 players

- Sequential: first FM, then SM

- 'No unconditional cooperation' 


\section{The game}

- Prisoner Dilemma variant, 2 players

- Sequential: first FM, then SM

- 'No unconditional cooperation'

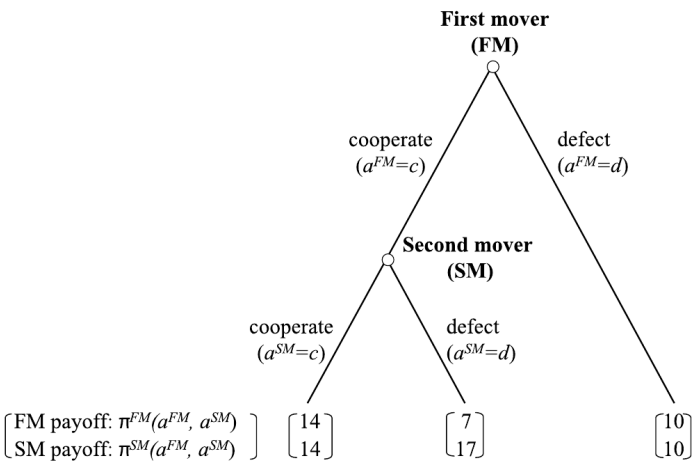




\section{The experiment}

- Each participants plays both roles: first SM, then FM. Paired randomly 


\section{The experiment}

- Each participants plays both roles: first SM, then FM. Paired randomly

- Different conditions:

$\rightarrow$ "How many opponents did, according to you, cooperate?"

$\rightarrow$ Feedback 


\section{The experiment}

- Each participants plays both roles: first SM, then FM. Paired randomly

- Different conditions:

$\rightarrow$ "How many opponents did, according to you, cooperate?"

$\rightarrow$ Feedback

\begin{tabular}{r|lll}
\hline Treatment & Baseline & Elicit_Beliefs & True_Distribution \\
\hline \hline Task 1 & 2nd move & 2nd move & 2nd move \\
Feedback $\left(a_{-i}^{S M}\right)$ & No & No & Yes \\
Task 2 & 1st move & beliefs $\left(a_{-i}^{S M}\right)$ & 1st move \\
Task 3 & beliefs $\left(a_{-i}^{F M}\right)$ & 1st move & beliefs $\left(a_{-i}^{F M}\right)$ \\
\hline N. Participants & 40 & 60 & 60 \\
\hline
\end{tabular}




\section{FM and SM correlation}

FMCR and SMCR 


\section{FM and SM correlation}

\section{FMCR and SMCR}

- Positive correlation

- Significant in all conditions

- Discussed in original paper 


\section{Consensus effect}

SMCR and beliefs 


\section{Consensus effect}

\section{SMCR and beliefs}

- Beliefs are biased towards actions (driven by preferences)

- Focus of original paper

- Seen as social projection by Busemeyer \& Pothos (2012), suitable for a quantum model 


\section{Reasoned player}

\section{Beliefs and FMCR}




\section{Reasoned player}

\section{Beliefs and FMCR}

- Forcing a player to form an opinion, changes the player 


\section{Reasoned player}

\section{Beliefs and FMCR}

- Forcing a player to form an opinion, changes the player

- Seen in an order effect

\begin{tabular}{r|llll}
\hline Treatment & Baseline & Elicit_Beliefs & True_Distribution & Total \\
\hline \hline First mover (FM) & $27.5 \%$ & $55.0 \%$ & $56.7 \%$ & $48.8 \%$ \\
Second mover (SM) & $55.0 \%$ & $53.3 \%$ & $55.0 \%$ & $54.4 \%$ \\
\hline
\end{tabular}




\section{Reasoned player}

\section{Beliefs and FMCR}

- Forcing a player to form an opinion, changes the player

- Seen in an order effect

\begin{tabular}{r|llll}
\hline Treatment & Baseline & Elicit_Beliefs & True_Distribution & Total \\
\hline \hline First mover (FM) & $27.5 \%$ & $55.0 \%$ & $56.7 \%$ & $48.8 \%$ \\
Second mover (SM) & $55.0 \%$ & $53.3 \%$ & $55.0 \%$ & $54.4 \%$ \\
\hline
\end{tabular}

- Equivalent to full information

- Measurement influences the system 


\section{The measurements}

- FM measurement $\rightarrow\left\{\left|a_{C}^{F M}\right\rangle,\left|a_{D}^{F M}\right\rangle\right\}$

- SM measurement $\rightarrow\left\{\left|a_{C}^{F M}\right\rangle,\left|a_{D}^{F M}\right\rangle\right\}$ 


\section{The measurements}

- FM measurement $\rightarrow\left\{\left|a_{C}^{F M}\right\rangle,\left|a_{D}^{F M}\right\rangle\right\}$

- SM measurement $\rightarrow\left\{\left|a_{C}^{F M}\right\rangle,\left|a_{D}^{F M}\right\rangle\right\}$

- Belief measurement $\rightarrow\{|?\rangle\}$ 


\section{The measurements}

- FM measurement $\rightarrow\left\{\left|a_{C}^{F M}\right\rangle,\left|a_{D}^{F M}\right\rangle\right\}$

- SM measurement $\rightarrow\left\{\left|a_{C}^{F M}\right\rangle,\left|a_{D}^{F M}\right\rangle\right\}$

- Belief measurement $\rightarrow\{|?\rangle\}$

- Build the Hilbert Space by modeling the three effects 


\section{Three effects revisited}

FM \& SM correlation: 


\section{Three effects revisited}

FM \& SM correlation:

- Classical correlation, can be measured at the same time

- In $\mathbb{H}^{4}$ spanned $\left\{\left|a_{i}^{F M}\right\rangle \otimes\left|a_{j}^{S M}\right\rangle\right\}$

- Not unlike Pothos and Busemeyer (2009) 


\section{Three effects revisited}

\section{Consensus effect:}




\section{Three effects revisited}

\section{Consensus effect:}

- SM and beliefs are complementary measurements.

- Different bases in the Hilbert space $\mathbb{H}^{C E}$. 


\section{Three effects revisited}

Consensus effect:

- SM and beliefs are complementary measurements.

- Different bases in the Hilbert space $\mathbb{H}^{C E}$.

- Form of Social Projection. (Busemeyer \& Pothos (2012)) 


\section{Three effects revisited}

Consensus effect:

- SM and beliefs are complementary measurements.

- Different bases in the Hilbert space $\mathbb{H}^{C E}$.

- Form of Social Projection. (Busemeyer \& Pothos (2012))

Reasoned player: 


\section{Three effects revisited}

Consensus effect:

- SM and beliefs are complementary measurements.

- Different bases in the Hilbert space $\mathbb{H}^{C E}$.

- Form of Social Projection. (Busemeyer \& Pothos (2012))

Reasoned player:

- FM and beliefs are complementary measurements.

- Different bases in the Hilbert space $\mathbb{H}^{R P}$. 


\section{Three effects revisited}

Consensus effect:

- SM and beliefs are complementary measurements.

- Different bases in the Hilbert space $\mathbb{H}^{C E}$.

- Form of Social Projection. (Busemeyer \& Pothos (2012)) Reasoned player:

- FM and beliefs are complementary measurements.

- Different bases in the Hilbert space $\mathbb{H}^{R P}$.

- Forming the belief opinion changes the player, seen in the order effect 


\section{Three effects revisited}

Consensus effect:

- SM and beliefs are complementary measurements.

- Different bases in the Hilbert space $\mathbb{H}^{C E}$.

- Form of Social Projection. (Busemeyer \& Pothos (2012))

Reasoned player:

- FM and beliefs are complementary measurements.

- Different bases in the Hilbert space $\mathbb{H}^{R P}$.

- Forming the belief opinion changes the player, seen in the order effect

Tensoring the $\mathrm{SM}$ and $\mathrm{FM}$ bases of $\mathbb{H}^{C E}$ and $\mathbb{H}^{R P}$ gives us the required Hilbert Space $\mathbb{H}^{C E} \otimes \mathbb{H}^{R P}$. 


\section{Three effects revisited}

Consensus effect:

- SM and beliefs are complementary measurements.

- Different bases in the Hilbert space $\mathbb{H}^{C E}$.

- Form of Social Projection. (Busemeyer \& Pothos (2012))

Reasoned player:

- FM and beliefs are complementary measurements.

- Different bases in the Hilbert space $\mathbb{H}^{R P}$.

- Forming the belief opinion changes the player, seen in the order effect

Tensoring the $\mathrm{SM}$ and $\mathrm{FM}$ bases of $\mathbb{H}^{C E}$ and $\mathbb{H}^{R P}$ gives us the required Hilbert Space $\mathbb{H}^{C E} \otimes \mathbb{H}^{R P}$.

This tensoring also defines the belief base of $\mathbb{H}^{C E} \otimes \mathbb{H}^{R P}$. 


\section{Dimension of the belief basis?}

- Belief measurement has 10 possible outcomes $\rightarrow$ dimension too high 


\section{Dimension of the belief basis?}

- Belief measurement has 10 possible outcomes $\rightarrow$ dimension too high

- 'estimates the probability a player thinks his opponent will cooperate or defect' 


\section{Dimension of the belief basis?}

- Belief measurement has 10 possible outcomes $\rightarrow$ dimension too high

- 'estimates the probability a player thinks his opponent will cooperate or defect' (does this work?)

$\rightarrow$ Beliefbasis (and $\mathbb{H}^{C E}$ and $\mathbb{H}^{R P}$ ) 2 dimensional 


\section{Dimension of the belief basis?}

- Belief measurement has 10 possible outcomes $\rightarrow$ dimension too high

- 'estimates the probability a player thinks his opponent will cooperate or defect' (does this work?)

$\rightarrow$ Beliefbasis (and $\mathbb{H}^{C E}$ and $\mathbb{H}^{R P}$ ) 2 dimensional

- EX: Player thinks 7 opponents cooperate:

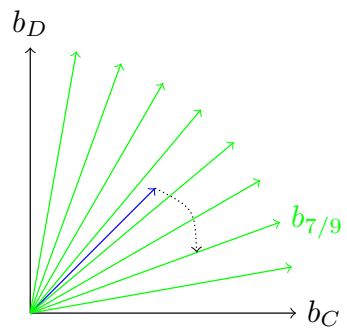




\section{QP\&B model}

- Player is represented by a state vector in $\mathbb{H}^{4}=\mathbb{H}^{2} \otimes \mathbb{H}^{2}$, spanned by $\left\{\left|a_{i}^{F M}\right\rangle \otimes\left|a_{j}^{S M}\right\rangle\right\}$. 


\section{QP\&B model}

- Player is represented by a state vector in $\mathbb{H}^{4}=\mathbb{H}^{2} \otimes \mathbb{H}^{2}$, spanned by $\left\{\left|a_{i}^{F M}\right\rangle \otimes\left|a_{j}^{S M}\right\rangle\right\}$.

- FM action is asscoiated with the planes $\left|a_{C}^{F M}\right\rangle \otimes\left|a_{j}^{S M}\right\rangle$ and $\left|a_{D}^{F M}\right\rangle \otimes\left|a_{j}^{S M}\right\rangle$.

- $\mathrm{SM}$ action is asscoiated with the planes $\left|a_{i}^{F M}\right\rangle \otimes\left|a_{C}^{S M}\right\rangle$ and $\left|a_{j}^{F M}\right\rangle \otimes\left|a_{D}^{S M}\right\rangle$. 


\section{QP\&B model}

- Player is represented by a state vector in $\mathbb{H}^{4}=\mathbb{H}^{2} \otimes \mathbb{H}^{2}$, spanned by $\left\{\left|a_{i}^{F M}\right\rangle \otimes\left|a_{j}^{S M}\right\rangle\right\}$.

- FM action is asscoiated with the planes $\left|a_{C}^{F M}\right\rangle \otimes\left|a_{j}^{S M}\right\rangle$ and $\left|a_{D}^{F M}\right\rangle \otimes\left|a_{j}^{S M}\right\rangle$.

- $S M$ action is asscoiated with the planes $\left|a_{i}^{F M}\right\rangle \otimes\left|a_{C}^{S M}\right\rangle$ and $\left|a_{j}^{F M}\right\rangle \otimes\left|a_{D}^{S M}\right\rangle$.

- In $\mathbb{H}^{4}$ we have 2 orthogonal planes $B_{C}$ en $B_{D}$.

- Bundle of planes spanned by $B_{C}$ and $B_{D}$, contains the planes associated with belief measurement. 


\section{Discussion and future plans}

- Correlation/proportions of FMCR and SMCR are modeled within the state vector 


\section{Discussion and future plans}

- Correlation/proportions of FMCR and SMCR are modeled within the state vector

- Relation between beliefs and actions are modeled by the angles between the beliefplanes and actionplanes. 


\section{Discussion and future plans}

- Correlation/proportions of FMCR and SMCR are modeled within the state vector

- Relation between beliefs and actions are modeled by the angles between the beliefplanes and actionplanes.

- Fit is promising:

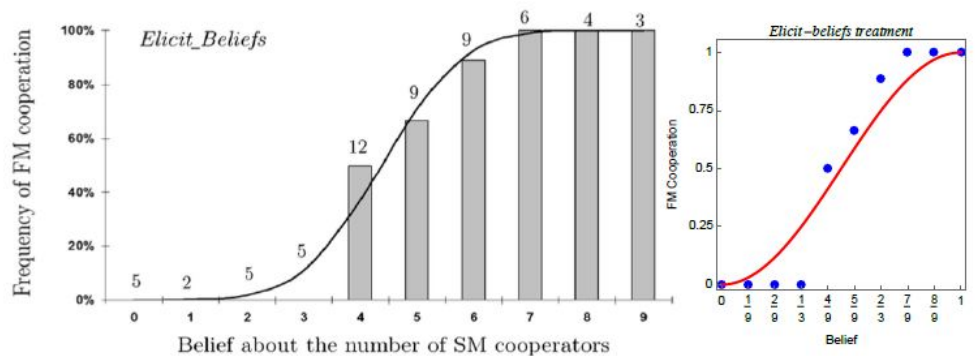

